\title{
REGISTROS GRÁFICO E ALGÉBRICO EM ATIVIDADES SOBRE FUNÇÕES QUADRÁTICAS: UM ESTUDO POR MEIO DO APLICATIVO GEOGEBRA EM SMARTPHONES
}

\section{GRAPHIC AND ALGEBRAIC RECORDS IN ACTIVITIES ON QUADRATIC FUNCTIONS: A STUDY THROUGH THE GEOGEBRA APPLICATION IN SMARTPHONES}

\author{
Wellington Fernando Delvechio Gama Garcia \\ Universidade Estadual do Paraná - UNESPAR \\ wellingtondelvechio@gmail.com \\ Lisiane Cristina Amplatz \\ Universidade Estadual do Oeste do Paraná - UNIOESTE \\ lisianeca@gmail.com \\ Veridiana Rezende \\ Universidade Estadual do Paraná - UNESPAR \\ rezendeveridiana@gmail.com
}

\begin{abstract}
Resumo
Este trabalho traz resultados de uma investigação que teve como objetivo principal investigar as resoluções de estudantes do $1^{\circ}$ ano do Ensino Médio relativas a atividades de função quadrática, envolvendo a conversão entre os registros de representação simbólico algébrico e gráfico, com o uso do aplicativo GeoGebra em smartphones. Para o desenvolvimento da pesquisa, foi realizada a aplicação de quatro blocos de atividades sobre função quadrática, com foco nas conversões entre os registros algébrico e gráfico, em uma turma de $1^{\circ}$ Ano do Ensino Médio de uma escola pública do Paraná. Para a resolução das atividades, os estudantes organizaram-se em dez duplas, um quarteto, e dois estudantes que optaram pela realização individual. Todos os estudantes utilizaram seus próprios smartphones. A organização/seleção das atividades e as análises das produções escritas dos estudantes ocorreram à luz da Teoria dos Registros de Representação Semiótica, de Raymond Duval. Ao final das análises, concluímos que os estudantes indicaram compreender a relação entre cada variável visual do gráfico com a sua correspondente unidade significativa da expressão algébrica, além do avanço nas notações e produções matemáticas escritas, percebido no decorrer da resolução das atividades.
\end{abstract}

Palavras-chave: Função Quadrática. Teoria dos Registros de Representação Semiótica. GeoGebra. Smartphone.

\begin{abstract}
This work brings results from an investigation which had as main objective investigating the resolutions by freshmen through quadratic function activities, involving the conversion between algebraic and graphic symbolic representation registers using the application GeoGebra on smartphones. For research development, the application of four blocks of activities on quadratic function were carried out, focused on the conversions between the algebraic and graphic records
\end{abstract}


in a freshmen class of a public school in Paraná. To solve the activities, students were organized in ten doubles and a quartet, and two students chosen the individual solution. All the students used their own smartphones. The organization/ selection of activities and the analysis of students' written productions occurred in the light of Theory of Semiotic Representation Records by Raymond Duval. At the end of the analyzes, we concluded that the students were able to understand the relationship between each visual variable in the graph with its corresponding significant unit of algebraic expression, further the advance in written mathematical notations and productions observed during the resolution of activities.

Keywords: Quadratic function. Theory of Semiotic Representation Records. GeoGebra. Smartphone.

\section{INTRODUÇÃO}

Indicativos de funções foram percebidos desde a Época Antiga, recebendo diversas contribuições de cientistas e filósofos ao longo da história, até se constituir como conceito matemático. Segundo Nogueira (2014, p. 1, grifo da autora), esse conceito tem origem em problemas práticos, permitindo explicar situações com base na realidade, tais como “[...] o movimento dos corpos, a vaporização da água, a germinação de uma semente $[\ldots]$ fenômenos que relacionam 'causa-efeito', ou em linguagem matemática, a dependência entre variáveis". Para a referida pesquisadora, são as funções que dão mobilidade à Matemática. Nesse sentido, diversos pesquisadores apontam o conceito de função como um dos mais importantes na Matemática (FONSECA; SANTOS; NUNES, 2013).

No entanto, pesquisas (SCANO, 2009; REIS, 2011; QUEIROZ, 2014; ROMANELLO, 2016) apontam para dificuldades na compreensão do conceito de função por estudantes da Educação Básica, e até mesmo do Ensino Superior. Tais dificuldades são relacionadas à interpretação de situações que envolvem expressões algébricas ou generalização de problemas; diferenciação entre equação e função; interpretação e reconhecimento de gráficos de funções; variação entre as grandezas de um gráfico, entre outras.

Birgin (2012) constatou que a maioria das dificuldades sobre função manifestadas pelos sujeitos de sua pesquisa provém do fato de que eles não conseguem associar diferentes formas de representação desse conceito e, assim, podem não compreendê-lo de forma eficaz e global. $\mathrm{O}$ autor ainda enfatiza que a compreensão do conceito de função em apenas um tipo de representação não garante seu entendimento em outro tipo de representação. Segundo o pesquisador, os estudantes "[...] precisam ser capazes de 
compreender as informações presentes nos diferentes formatos, e executar transições entre as várias representações ${ }^{1 "}$ (BIRGIN, 2012, p. 141, tradução nossa).

Nessa mesma direção, Duval (2003) menciona que aprender matemática é diferente de aprender outras ciências, nas quais os fenômenos podem ser observados. $\mathrm{Na}$ matemática, o acesso aos objetos ocorre somente por meio de suas representações, fato que pode desencadear o paradoxo: "Como podemos não confundir um objeto e sua representação se não temos acesso a esse objeto a não ser por meio de sua representação?" (DUVAL, 2003, p. 21). Para contornar essa contradição, o referido pesquisador defende que a compreensão de objetos matemáticos ocorrerá a partir da articulação entre suas diferentes representações.

A partir das dificuldades dos estudantes em relação ao conceito de função já apontadas em pesquisas (SCANO, 2009; REIS, 2011; BIRGIN, 2012; QUEIROZ, 2014; ROMANELLO, 2016), e considerando a importância, do ponto de vista de Duval (2003; 2009), da articulação entre diferentes registros de representação semiótica, propusemonos a investigar resoluções de estudantes do $1^{\circ}$ ano do Ensino Médio mediante atividades de função quadrática, envolvendo a conversão entre os registros de representação simbólico algébrico e gráfico, com o uso do aplicativo GeoGebra em smartphones.

\section{ASPECTOS DA TEORIA DOS REGISTROS DE REPRESENTAÇÃO SEMIÓTICA}

No decorrer de suas pesquisas em psicologia cognitiva, desde os anos de 1970, Raymond Duval defende que não há estudo de fenômenos relativos ao conhecimento sem recorrer às representações, pois “[...] não há conhecimento que não possa ser mobilizado por um sujeito sem uma atividade de representação" (DUVAL, 2009, p. 29). Em matemática, o autor considera que as representações semióticas são indispensáveis para a comunicação e necessárias para o desenvolvimento da atividade matemática.

A noção de representação foi tomada como representação semiótica a partir dos estudos sobre a aquisição dos conhecimentos matemáticos e sobre os problemas que sua aprendizagem poderia originar. A especificidade das representações semióticas

[...] consiste em serem relativas a um sistema particular de signos, a linguagem, a escrita algébrica ou os gráficos cartesianos, e podem ser convertidas em representações equivalentes em um outro sistema semiótico, mas podendo tomar significações diferentes para o sujeito que as utiliza (DUVAL, 2009, p. $32)$.

\footnotetext{
1 “[...] Students need to be able to understand information presented in these different formats and to perform transitions among the various representations" (BIRGIN, 2012, p. 141).
} 
Atualmente, há maior exigência na formação dos alunos, a fim de prepará-los para um mundo onde a tecnologia está cada vez mais complexa. Para tanto, Duval (2003, p. 11) salienta a necessidade de uma abordagem cognitiva,

[...] pois o objetivo do ensino de matemática, em formação inicial, não é formar futuros matemáticos, nem dar aos alunos instrumentos que só lhes serão eventualmente úteis muito mais tarde, e sim contribuir para o desenvolvimento geral de suas capacidades de raciocínio, de análise e de visualização.

Segundo o pesquisador, a originalidade de uma abordagem cognitiva "[...] não está em partir dos erros para tentar determinar as concepções dos alunos e a origem de suas dificuldades em álgebra” (DUVAL, 2003, p. 12), por exemplo, mas está em descrever o funcionamento cognitivo que permita ao aluno " $[. .$.$] compreender, efetuar e$ controlar ele próprio a diversidade dos processos matemáticos que lhe são propostos em situação de ensino" (DUVAL, 2003, p. 12).

Quanto ao ensino de função, a Base Nacional Comum Curricular - BNCC (BRASIL, 2018) argumenta de forma positiva sobre a utilização de diferentes registros de representação no estudo deste conceito, tanto no Ensino Fundamental como no Ensino Médio. Ainda segundo o documento, o trânsito entre os diversos registros de representação permite maior flexibilidade e fluidez na área, bem como favorece o desenvolvimento do raciocínio.

$\mathrm{Na}$ Matemática, há uma variedade de representações. $\mathrm{O}$ enunciado de um problema, por exemplo, pode ser representado em língua natural, e suas resoluções podem vir na forma de cálculos algébricos, numéricos, gráficos e figuras. Duval (2003) classifica quatro grandes tipos de Registros de Representação Semiótica: Linguagem Natural $^{2}$ (associações verbais, conceituais, forma de raciocinar); Sistemas de Escritas - Simbólico (numéricas, algébricas); Figuras Geométricas, planas ou em perspectivas (operação operatória e não somente discursiva, construção com instrumentos); e Gráficos Cartesianos (mudanças de sistemas de coordenadas, interpolação, extrapolação).

Ao reconhecer diferentes representações de um mesmo objeto matemático, o estudante pode optar pelo modo mais eficaz para resolver uma atividade proposta. Assim, ele pode utilizar seus conhecimentos para resolver da forma mais rápida e prática, pois “[...] tendo mais registros, há um aumento potencial de possibilidades de trocas e, por conseguinte, há um aumento também na escolha mais econômica” (MORETTI, 2002, p. 346).

\footnotetext{
${ }^{2}$ No Brasil, entendemos por linguagem natural a escrita em língua portuguesa - língua ofícial do país.
} 
Duval (2009) considera essencial, para a aprendizagem de um conceito, dois tipos de transformações - o tratamento e a conversão. Por tratamento, o pesquisador define como "[...] uma transformação de representação interna a um registro de representação" (DUVAL, 2009, p. 57); em outros termos, mesmo após a realização da transformação do objeto matemático, mantém-se o registro inicial. Podemos observar um tratamento no registro de representação algébrico, por exemplo, na determinação da lei geral de uma função quadrática a partir da sua forma fatorada, ou seja,

$$
\begin{gathered}
f(x)=(x+3) \cdot(x+2) \\
f(x)=x^{2}+2 \cdot x+3 \cdot x+6 \\
f(x)=x^{2}+5 \cdot x+6 .
\end{gathered}
$$

Já conversão é “[...] transformar a representação de um objeto ou de uma informação dada num registro em uma representação desse mesmo objeto, dessa mesma situação ou da mesma informação num outro registro" (DUVAL, 2009, p. 58), logo, é uma transformação externa ao registro de partida. Como exemplo de conversão, podemos citar um problema dado em língua natural, o qual necessita de transformação para o registro algébrico para que possa ser solucionado.

\begin{tabular}{l|c}
\multicolumn{1}{c|}{ Registro em língua natural } & $\begin{array}{c}\text { Conversão para o registro algébrico de uma } \\
\text { equação quadrática }\end{array}$ \\
\hline $\begin{array}{l}\text { Os 180 alunos de uma escola estão dispostos em } \mathrm{x} \\
\text { filas, de tal modo que o número de alunos de cada } \\
\text { fila supera em } 8 \text { o número de filas. Quantos alunos } \\
\text { há em cada fila? (Adaptado de Dante (2017)). }\end{array}$ & $x \cdot(x+8)=180$ \\
\end{tabular}

Neste caso, os registros de representação da equação quadrática são diferentes, mas dizem respeito ao mesmo objeto matemático. Além disso, as representações não constituem todo o conceito de um objeto matemático. Como exemplo, Moretti menciona que a:

[...] linguagem discursiva não oferece as mesmas possibilidades que podem oferecer uma figura ou um diagrama. Isto quer dizer que de um ponto de vista cognitivo uma representação é parcial em relação aquilo que ela quer representar e que de um registro a outro não são os mesmos conteúdos de uma situação que são representados (MORETTI, 2002, p. 347).

Na transformação de conversão, Duval (2003) estabelece a existência de dois tipos de fenômenos, a saber: A) as variações de congruência e de não-congruência; e B) a heterogeneidade dos dois sentidos de conversão. Quando analisamos a resolução de uma tarefa em que houve uma atividade de conversão, duas situações podem ocorrer, ao 
compararmos a representação no registro de partida com a representação terminal no registro de chegada:

[...] ou a representação terminal transparece na representação de saída e a conversão está próxima de uma situação de simples codificação - diz-se então que há congruência -, ou ela não transparece absolutamente e se dirá que ocorre a não-congruência [sic] (DUVAL, 2003, p. 19).

Para Duval (2012), quando há congruência entre os registros de partida e chegada, a conversão é trivial, comparada intuitivamente a um simples código. No entanto, quando há não congruência, a conversão pode se tornar custosa em relação ao tempo de tratamento, e pode "[...] criar um problema diante do qual o sujeito se sente desarmado e a possibilidade de conversão não vem mais à mente" (DUVAL, 2012, p. 284).

Dentre as dificuldades apresentadas pelos estudantes, com vistas ao conceito de função, destacamos aquelas citadas por Duval (2011), associadas aos registros de representação semiótica: simbólico algébrico e gráfico. Isto porque, no ensino, “[...] atém-se a passagem da equação para a sua representação gráfica com a construção ponto a ponto, esquece-se que é a passagem inversa que traz problema" (DUVAL, 2011, p. 97). A maioria das atividades pedagógicas, sejam de livros didáticos ou materiais afins, está voltada para a correspondência entre da representação algébrica para a representação gráfica, sem operar o processo inverso, tornando as representações gráficas “[...] obscuras para a maioria dos alunos" (DUVAL, 2011, p. 98).

Nesse sentido, Lucena e Gitirana (2016, p. 25) afirmam que o “[...] que se percebe, porém, é um ensino que prioriza o tratamento algébrico e deixa a desejar a interpretação geométrica do objeto matemático representado, o que dificulta um aprofundamento maior dos conceitos em foco".

Duval (2011) explica que há três abordagens possíveis para a representação gráfica, e elas não operam com os mesmos dados visuais do gráfico. A abordagem ponto a ponto, mais comum entre as atividades pedagógicas, é utilizada para introduzir a construção gráfica a partir da associação de um par de números a um ponto no gráfico, e vice-versa. Segundo o pesquisador, essa estratégia não permite boa interpretação do gráfico, e pode causar problemas quando se trata de converter do registro Gráfico para o registro Simbólico Algébrico, pois se detém apenas nos pontos determinados, e não no conceito de função em si.

A abordagem de extensão do traçado efetuado refere-se a uma ampliação da abordagem ponto a ponto, pois não se limita aos pontos determinados previamente, referindo-se a um conjunto infinito de pontos em potencial. Essas duas abordagens 
consideram, prioritariamente, os dados do traçado, e “[...] não as variáveis visuais pertinentes da representação gráfica” (DUVAL, 2011, p. 99). Assim, “[...] o tratamento se mantém orientado na busca de valores particulares sem se ocupar com a forma da expressão algébrica” (DUVAL, 2011, p. 99).

Em atividades cujo objetivo é partir da representação gráfica para a representação algébrica correspondente e vice e versa, Duval (2011) privilegia a abordagem de interpretação global de propriedades figurais. Em outras palavras, é uma abordagem que requer um trabalho de congruência entre os dois registros de apresentação de um objeto e, nesse caso, um trabalho envolvendo a representação gráfica e algébrica de uma função, concomitantemente. Para o pesquisador, a

[...] prática sistemática da abordagem ponto a ponto não favorece a abordagem de interpretação global que é em geral deixada de lado no ensino uma vez que depende de análise semiótica visual e algébrica. Compreende-se por que a maioria dos alunos fica aquém de uma utilização correta das representações gráficas (DUVAL, 2011, p. 99).

Para Moretti (2003), a interpretação global de propriedades figurais permite ao aluno identificar as modificações possíveis na imagem gráfica e na expressão algébrica conjuntamente. Para Duval (2011), nesse tipo de abordagem se busca perceber a associação de uma variável visual de representação com sua unidade significativa da expressão algébrica, prática que não ocorre nas abordagens ponto a ponto e de extensão do traçado efetuado, visto que estas últimas desviam a atenção dos estudantes para as variáveis visuais.

As unidades significativas em uma expressão algébrica correspondem a cada um dos símbolos envolvidos na expressão, por exemplo: $>$ (maior que), $<$ (menor que), = (igual a), símbolo da variável, símbolos de operações e sinais + (soma), - (subtração), dentre outros. O objetivo central dessa abordagem é "[...] corresponder variáveis visuais pertinentes do gráfico com unidades significativas da expressão algébrica” (DUVAL, 2011, p. 100).

Em relação à função quadrática em sua forma canônica ${ }^{3}$, ou seja, $f(x)=a(x+m)^{2}$ $+n$, sendo $a, m$ e $n$ números reais, e a interpretação global de propriedades figurais, Maia (2007) apresenta quatro variáveis visuais relacionadas à representação gráfica da função, associadas a suas respectivas unidades significativas da expressão algébrica da função, conforme quadro 1 .

\footnotetext{
${ }^{3}$ A forma canônica da função quadrática deixa claro, ao contrário da forma estendia $f(x)=a x^{2}+b x+c$, os pontos do vértice da função. Ela é da forma $f(x)=(a x-m)^{2}+k$.
} 
Garcia, W. F. D. G.; Amplatz, L. C.; Rezende, V.

Quadro 1 - Variáveis visuais e as respectivas unidades simbólicas significativas da função quadrática

\begin{tabular}{|c|c|c|}
\hline Variáveis Visuais & Valores & $\begin{array}{c}\text { Unidades simbólicas } \\
\text { correspondente }\end{array}$ \\
\hline Concavidade da parábola & $\begin{array}{l}\text { Voltada para cima } \\
\text { Voltada para baixo }\end{array}$ & $\begin{array}{l}a>0 \\
a<0\end{array}$ \\
\hline Abertura da parábola & $\begin{array}{l}\text { Maior abertura } \\
\text { Menor abertura }\end{array}$ & $\begin{array}{l}0<|a|<1 \\
|a|>1\end{array}$ \\
\hline $\begin{array}{l}\text { Posição do vértice da parábola com } \\
\text { relação ao eixo das abscissas }\end{array}$ & $\begin{array}{l}\text { Acima do eixo } \\
\text { Na origem } \\
\text { Abaixo do eixo }\end{array}$ & $\begin{array}{l}\mathrm{n}>0 \\
\mathrm{n}=0 \\
\mathrm{n}<0\end{array}$ \\
\hline $\begin{array}{l}\text { Posição do vértice da parábola com } \\
\text { relação ao eixo das ordenadas }\end{array}$ & $\begin{array}{l}\text { A esquerda do eixo } \\
\mathrm{Na} \text { origem } \\
\mathrm{A} \text { direita do eixo }\end{array}$ & $\begin{array}{l}\mathrm{m}>0 \\
\mathrm{~m}=0 \\
\mathrm{~m}<0\end{array}$ \\
\hline
\end{tabular}

Fonte: Autores com base em Maia (2007).

Neste contexto do uso de diferentes registros de representação semiótica, especialmente com o foco na interpretação global das propriedades figurais para $\mathrm{o}$ conceito de função quadrática, para a presente pesquisa, consideramos o uso do software GeoGebra como ferramenta de apoio para o desenvolvimento das atividades pelos estudantes. Entendemos que este recurso, além de possibilitar a construção dos gráficos com mais agilidade, ele "[...] permite o uso de representações distintas do mesmo objeto matemático, de forma dinâmica e articulada, possibilitando ao usuário a visualização de tais representações em uma única tela" (LUCENA; GITIRANA, 2016, p. 27). O Geogebra possui, ainda, duas janelas simultâneas, uma de álgebra e uma gráfica, e com isso, Lucena e Gitirana (2016, p. 27) defendem que se trata de “[...] um recurso importante para uma abordagem do conceito de parábola que busque articular os dois subcampos da matemática, como também, que articule diferentes representações".

Por fim, levando em consideração os direcionamentos dados pela BNCC (BRASIL, 2018) a respeito do ensino de função e o aporte teórico dos Registros de Representação Semiótica de Raymond Duval apresentados neste texto, propusemos a estudantes do Ensino Médio atividades relacionadas à função quadrática com o propósito de articular suas representações algébrica e gráfica. Utilizamos, para isso, o aplicativo para smartphone GeoGebra para auxiliar e otimizar o tempo de construção de diversos gráficos pelos estudantes colaboradores desta pesquisa.

As atividades propostas foram consideradas com a intenção de contemplar a abordagem de interpretação global das propriedades figurais (DUVAL, 2011), para que os estudantes fossem capazes de interpretar e relacionar cada unidade significativa do registro simbólico algébrico às respectivas variáveis visuais no registro gráfico. Para isso, apresentamos, na seção a seguir, os procedimentos metodológicos utilizados na trajetória desta pesquisa. 


\section{PROCEDIMENTOS METODOLÓGICOS}

Para o desenvolvimento desta pesquisa, tomamos como base as atividades propostas na pesquisa de Mestrado de Maia (2007), que teve como sujeitos estudantes do $9^{\circ}$ ano da cidade de São Paulo, com o objetivo de explicitar o que cada variação nas unidades significativas na expressão algébrica de uma função quadrática pode implicar no registro gráfico, utilizando o software gráfico Winplot, no ambiente de laboratório de informática.

Elucidamos que a intenção da presente investigação não foi a de realizar uma comparação com os resultados da pesquisa de Maia (2007), mas apenas tomar respaldo em seu instrumento de pesquisa e, a partir de adaptações, produzir e analisar os dados desta pesquisa.

Sendo assim, a partir da sequência didática elaborada por Maia (2007), fizemos adaptações necessárias conforme o contexto da escola envolvida na pesquisa, a quantidade de horas disponíveis para a aplicação das atividades em sala de aula, o uso do GeoGebra via Smartphones e o foco deste trabalho, conforme apresentado a seguir. As adaptações na sequência didática ocorreram conforme informações disponibilizadas no quadro 2.

Quadro 2 - Adaptações da sequência didática da Maia (2007) para a realização desta pesquisa

\begin{tabular}{|c|c|}
\hline Pesquisa de Maia (2007) & Presente pesquisa \\
\hline $\begin{array}{l}\text { - Turma: } 8^{\text {a }} \text { série (atual } 9^{\circ} \text { ano) do Ensino } \\
\text { Fundamental; } \\
\text { - Pesquisa desenvolvida em horário contrário às } \\
\text { aulas regulares; } \\
\text { - } 8 \text { alunos participantes da pesquisa; } \\
\text { - Atividades implementadas no laboratório de } \\
\text { informática da escola. }\end{array}$ & $\begin{array}{l}\text { - Turma: } 1^{\circ} \text { ano do Ensino Médio; } \\
\text { - Pesquisa desenvolvida em horário regular de } \\
\text { aula, durante a regência na disciplina de Estágio } \\
\text { Supervisionado II pelo primeiro autor; } \\
\text { - } 26 \text { alunos participantes da pesquisa. }\end{array}$ \\
\hline $\begin{array}{l}\text { Atividades resolvidas pelos alunos nos } \\
\text { computadores do laboratório. }\end{array}$ & $\begin{array}{l}\text { Atividades resolvidas pelos alunos em seus } \\
\text { próprios smartphones. }\end{array}$ \\
\hline $\begin{array}{l}\text { Atividades resolvidas com o auxílio do software } \\
\text { Winplot. }\end{array}$ & $\begin{array}{l}\text { Atividades resolvidas com o auxílio do aplicativo } \\
\text { GeoGebra. }\end{array}$ \\
\hline Sequência de seis atividades. & $\begin{array}{l}\text { Atividades 1, } 2 \text { e } 3 \text { de Maia (2007), com } \\
\text { adaptações. }\end{array}$ \\
\hline
\end{tabular}

Fonte: Elaborado pelos autores.

Ainda em relação às adaptações realizadas na sequência didática de Maia (2007), duas atividades não foram contempladas, pois não foram foco da presente pesquisa. A primeira solicitava aos estudantes estabelecerem uma relação algébrica entre os coeficientes da função quadrática em sua forma canônica, a qual consideramos não atender às expectativas da professora regente da turma, por não fazer parte de seu planejamento de ensino. A segunda tratava da construção de duas máscaras teatrais, das quais o contorno eram parábolas. Consideramos que estas construções demandariam 
bastante tempo em sala de aula, além de o estudante necessitar a compreensão do domínio e imagem da função quadrática.

A partir das atividades 1, 2 e 3 de Maia (2007), novos itens foram acrescentados com outros valores para as funções em seus registros algébricos. Assim, por meio de diversas resoluções e com o auxílio do aplicativo GeoGebra para smartphone, os estudantes puderam realizar conversões para o registro gráfico e visualizar concomitantemente as relações entre as variáveis visuais e unidades significativas destes dois registros de representação.

Após as devidas alterações na sequência didática, o nosso instrumento de pesquisa consistiu-se em quatro (04) blocos de atividades, dos quais três (03) tratavam do coeficiente da função quadrática em sua forma canônica e, por último, uma (01) atividade sintetizava todas as informações abordadas nos três primeiros blocos.

As atividades foram aplicadas em uma turma do $1^{\circ}$ Ano do Ensino Médio do curso Técnico em Administração, a qual era a turma cujo primeiro autor deste artigo realizou a regência da Disciplina de Estágio Supervisionado II. No dia da implementação das atividades, 26 estudantes estavam presentes na aula, os quais se organizaram da seguinte forma: dez duplas, um quarteto, e dois estudantes optaram pela resolução individual.

No momento de implementação das atividades, contamos com auxílio de uma estagiária para as observações, e a professora regente permaneceu presente em sala, observando todo o processo. O desenvolvimento do trabalho em sala de aula ocorreu em quatro aulas de 50 minutos cada. Além das atividades impressas, os estudantes tiveram acesso aos materiais escolares básicos (lápis, caneta, régua e borracha) e ao aplicativo GeoGebra instalado em seus smartphones. Durante a implementação, fizemos anotações em um diário de campo com algumas falas dos estudantes, tanto durante a resolução quanto na correção das atividades. Os registros escritos dos estudantes foram recolhidos para análises.

Ao final das resoluções pelos estudantes, realizamos a formalização das respostas com toda a turma, momento em que discutimos os resultados alcançados por cada grupo e chegamos, durante essas discussões, às respostas matematicamente corretas para cada uma das atividades. 


\section{DESCRIÇÃO DAS ATIVIDADES E ANÁLISE DOS DADOS}

Esta sequência de atividades se mostrou eficiente em evidenciar, aos estudantes, cada variável visual da representação gráfica e sua respectiva unidade significativa da representação algébrica. As atividades desenvolvidas na presente pesquisa estão apresentadas no quadro 3.

Quadro 3 - Atividades

\section{Bloco de Atividades 1}

1. Num mesmo par de eixos cartesianos represente, utilizando o GeoGebra, o gráfico de:
a. $f_{1}(x)=x^{2}$
h. $f_{8}(x)=-x^{2}$
b. $f_{2}(x)=\frac{1}{2} x^{2}$
i. $f_{9}(x)=-\frac{1}{2} x^{2}$
c. $f_{3}(x)=5 x^{2}$
j. $f_{10}(x)=-5 x^{2}$
d. $f_{4}(x)=10 x^{2}$
k. $f_{11}(x)=-10 x^{2}$
e. $f_{5}(x)=\frac{1}{4} x^{2}$
I. $f_{12}(x)=-\frac{1}{4} x^{2}$
f. $f_{6}(x)=20 x^{2}$
m. $f_{13}(x)=20 x^{2}$
g. $f_{7}(x)=\frac{1}{20} x^{2}$
n. $f_{14}(x)=\frac{1}{20} x^{2}$

\section{Analisando os gráficos:}

a) O que é possível concluir a respeito do coeficiente de $\mathrm{x}^{2}$ ser um número maior que zero? E menor que zero?

b) O que é possível concluir quando o valor do coeficiente cresce?

c) Os gráficos possuem algum ponto em comum? Em caso positivo, indique o ponto.

d) Comparando os gráficos do exercício 1, o que se pode concluir?

\section{Bloco de Atividades 2}

1. Num mesmo par de eixos cartesianos represente, utilizando o GeoGebra, o gráfico de:
a. $f_{1}(x)=x^{2}$
f. $f_{6}(x)=x^{2}-1$
b. $f_{2}(x)=x^{2}+1$
g. $f_{7}(x)=x^{2}-2$
c. $f_{3}(x)=x^{2}+2$
h. $f_{8}(x)=x^{2}-5$
d. $f_{4}(x)=x^{2}+5$
i. $f_{9}(x)=x^{2}-10$
e. $f_{5}(x)=x^{2}+10$

2. Analisando os gráficos:

a) O que acontece com o gráfico da função quando se soma ou subtrai uma constante para obter uma nova função?

b) Quais são as coordenadas do vértice da parábola em cada um dos casos?

\section{Bloco de Atividades 3}

4. 1. Num mesmo par de eixos cartesianos represente, utilizando o GeoGebra, o gráfico de:
a. $f_{1}(x)=x^{2}$
f. $f_{6}(x)=(x-1)^{2}$
b. $f_{2}(x)=(x+1)^{2}$
g. $f_{7}(x)=\left(x-\frac{1}{2}\right)^{2}$
c. $f_{3}(x)=\left(x+\frac{1}{2}\right)^{2}$
h. $f_{8}(x)=(x-5)^{2}$
d. $f_{4}(x)=(x+5)^{2}$
i. $f_{9}(x)=\left(x-\frac{1}{10}\right)^{2}$
e. $f_{5}(x)=\left(x+\frac{1}{10}\right)^{2}$

2. Analisando os gráficos:

a) O que acontece com o gráfico conforme somamos ou subtraímos uma constante positiva da variável independente $\mathrm{x}$ ?

b) Quais são as coordenadas do vértice da parábola em cada um dos casos?

\section{Bloco de Atividades 4}

1. Você poderia dizer, baseado nas atividades anteriores, quais são as características do gráfico da função quadrática que são possíveis de notar na representação algébrica da função quadrática?

Fonte: Adaptado de Maia (2007). 


\section{Análise do Bloco de Atividades 1}

No bloco de atividades 1 , que diz respeito às variáveis visuais de representação concavidade e abertura da parábola, a partir da análise do sinal e do valor para o coeficiente $a$ da função quadrática, houve variedade de respostas apresentadas pelos estudantes. No item (a), dos treze (13) grupos de estudantes, nove (09) respostas foram corretas, três (03) respostas parcialmente corretas e uma (01) incorreta. Os estudantes compreenderam as implicações das modificações do coeficiente $a$ do registro simbólico algébrico da função quadrática para o registro gráfico. Como exemplo, citamos a resposta do Grupo 6, na figura 1, ao ser questionado sobre o fato de o coeficiente $a$ de $\mathrm{x}^{2}$ ser um número maior ou menor que zero.

Figura 1 - Resolução do Grupo 6

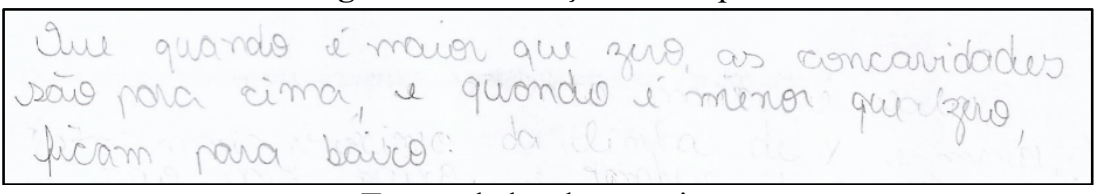

Fonte: dados da pesquisa.

Notamos, também, que a maioria dos estudantes utilizava termos que não eram corretos para representar o objeto em questão, conforme exemplo de resposta apresentada pelo Grupo 4: "Quando o gráfico é maior que 0, a reta vai para cima; quando é menor, a reta vai para baixo". Neste caso, os estudantes utilizaram equivocadamente o termo reta, pois o correto seria parábola. Ainda observamos a dificuldade do Grupo 4 em relação à escrita e ao emprego de termos matemáticos adequados. Apenas três (03) grupos utilizaram os termos parábola e concavidade para se referirem corretamente a esses objetos.

No item (b), nove (09) grupos apresentaram respostas corretas; dois (02) grupos, respostas parcialmente corretas; e outros dois (02) grupos, respostas incorretas. No caso das respostas parciais ou incorretas, os grupos relataram observações que não correspondiam à pergunta feita, como, por exemplo, a resposta do Grupo 1: "que ele é maior que zero".

O Grupo 10 apresentou como resposta: "O coeficiente pode dar positivo ou negativo, transformando ele [sic] para baixo ou para cima e infinito e conforme as parábolas". Significa que o grupo observou as transformações ocorridas no gráfico e suas observações foram corretas, mas não interpretou corretamente o questionamento do item (b), que se refere ao crescimento do coeficiente $a$ de $\mathrm{x}^{2}$. 
No que diz respeito ao item (c), sobre o ponto que todos os gráficos têm em comum, onze (11) respostas apresentadas pelos grupos foram consideradas parcialmente corretas; e duas (02), respostas incorretas. A maioria dos grupos identificou corretamente o ponto em comum, no caso a origem $(0,0)$, mas não apresentou o ponto em coordenada cartesiana, indicando como resposta apenas o "ponto zero". Este erro não ocorreu somente neste item, mas em outros também, nos quais foram solicitados a indicar os vértices da parábola.

O item (d) solicitava aos estudantes fazer uma síntese do que ocorre com o gráfico da função a partir de mudanças nos valores para o coeficiente $a$. Neste caso, os grupos deveriam associar as alterações do coeficiente $a$, ou seja, quando ele é positivo, negativo, quando se aproxima de zero, ou apresenta valores maiores, aos seus respectivos gráficos. Neste item, apenas três (03) respostas, apesar de serem parcialmente corretas, abordaram a maior parte das variáveis visuais ${ }^{4}$ do registro gráfico que estavam relacionadas ao coeficiente $a$. Por exemplo, a resposta do Grupo 8, na figura 2 .

Figura 2 - Resolução do Grupo $8^{5}$

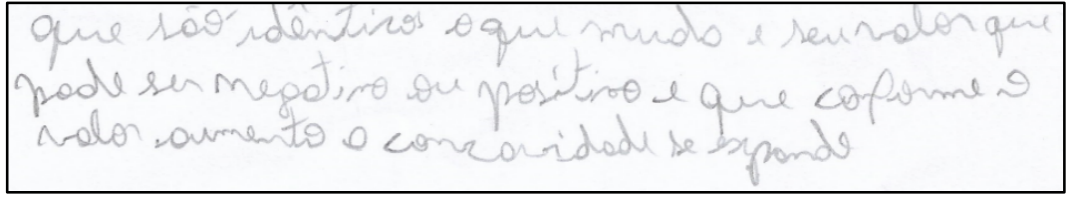

Fonte: dados da pesquisa.

Mesmo cometendo um erro em sua resposta (o correto seria dizer que a concavidade fecha), o Grupo 8 apresentou parte das variáveis visuais de representação que esperávamos ser listadas. O grupo mencionou sobre: a concavidade da parábola para cima ou para baixo, que está relacionada ao sinal do coeficiente $a$, positivo ou negativo; e a parábola abrir quando o valor do coeficiente $a$ fica pequeno, ou fechar quando ele fica grande. Em contrapartida, a maior parte dos grupos apresentou respostas incompletas, mencionando apenas sobre a variável visual concavidade da parábola, sem a associação com a unidade significativa da expressão algébrica, como, por exemplo, a resposta do Grupo 1, na figura 3.

Figura 3 - Resolução do Grupo 1

Tados eles parmam U cou $\cap$ (Paidsls)

Fonte: dados da pesquisa.

\footnotetext{
${ }^{4}$ Transladar o gráfico para cima ou para baixo, a abertura da parábola e transladar para a direita ou esquerda. 5 “que são idênticos, o que muda é seu valor, que pode ser negativo ou positivo, e que conforme o valor aumenta, a concavidade se expande" (Transcrição da escrita dos alunos do Grupo 8 na Figura 3).
} 
A seguir apresentamos, no quadro 4, um resumo das respostas dadas pelos alunos em cada item do bloco de atividades 1 (consideradas como correta (C), incorreta (I) ou parcial (P)), e em seguida, a classificação do erro dos alunos.

Quadro 4 - Resumo das respostas no bloco de atividades 1

\begin{tabular}{|c|c|c|c|c|c|}
\hline \multirow{2}{*}{ Grupo } & \multicolumn{4}{|c|}{$\begin{array}{l}\text { Situação por item } \\
(\mathrm{C}, \mathrm{P} \text { ou I })\end{array}$} & \multirow[t]{2}{*}{ Apontamentos } \\
\hline & (a) & (b) & (c) & (d) & \\
\hline 1 & $\mathrm{C}$ & I & $\mathrm{P}$ & $\mathrm{P}$ & $\begin{array}{l}\text { b) Não responde à pergunta; } \\
\text { c) Notação de coordenada; } \\
\text { d) Incompleta. }\end{array}$ \\
\hline 2 & $\mathrm{C}$ & $\mathrm{C}$ & $\mathrm{P}$ & $\mathrm{P}$ & $\begin{array}{l}\text { c) Notação de coordenada; } \\
\text { d) Incompleta. }\end{array}$ \\
\hline 3 & $\mathrm{P}$ & $\mathrm{C}$ & $\mathrm{P}$ & $\mathrm{P}$ & $\begin{array}{l}\text { c) Notação de coordenada; } \\
\text { d) Incompleta. }\end{array}$ \\
\hline 4 & $\mathrm{C}$ & $\mathrm{C}$ & $\mathrm{P}$ & I & $\begin{array}{l}\text { c) Notação de coordenada; } \\
\text { d) Não responde à pergunta. }\end{array}$ \\
\hline 5 & $\mathrm{C}$ & $\mathrm{C}$ & $\mathrm{P}$ & I & $\begin{array}{l}\text { c) Notação de coordenada; } \\
\text { d) Não responde à pergunta. }\end{array}$ \\
\hline 6 & $\mathrm{C}$ & $\mathrm{C}$ & $\mathrm{P}$ & $\mathrm{C}$ & c) Notação de coordenada. \\
\hline 7 & $\mathrm{C}$ & $\mathrm{C}$ & $\mathrm{P}$ & $\mathrm{P}$ & $\begin{array}{l}\text { c) Notação de coordenada; } \\
\text { d) Incompleta. }\end{array}$ \\
\hline 8 & I & $\mathrm{P}$ & I & $\mathrm{C}$ & $\begin{array}{l}\text { a) Não responde à pergunta; } \\
\text { c) Não responde à pergunta. }\end{array}$ \\
\hline 9 & $\mathrm{C}$ & $\mathrm{P}$ & I & $\mathrm{P}$ & c) Não responde à pergunta. \\
\hline 10 & $\mathrm{C}$ & I & $\mathrm{P}$ & I & $\begin{array}{l}\text { b) Não responde à pergunta; } \\
\text { c) Notação de coordenada; } \\
\text { d) Não responde à pergunta. }\end{array}$ \\
\hline 11 & $\mathrm{C}$ & $\mathrm{C}$ & $\mathrm{P}$ & $\mathrm{C}$ & c) Notação de coordenada; \\
\hline 12 & $\mathrm{P}$ & $\mathrm{C}$ & $\mathrm{P}$ & $\mathrm{P}$ & $\begin{array}{l}\text { c) Notação de coordenada; } \\
\text { d) Incompleta. }\end{array}$ \\
\hline 13 & $\mathrm{P}$ & $\mathrm{C}$ & $\mathrm{P}$ & $\mathrm{P}$ & $\begin{array}{l}\text { c) Notação de coordenada; } \\
\text { d) Incompleta. }\end{array}$ \\
\hline
\end{tabular}

Fonte: Autores.

\section{Análise do Bloco de Atividades 2}

No bloco de atividades 2, a respeito da variável visual de representação posição do vértice da parábola com relação ao eixo das ordenadas, notamos avanço nas respostas dos estudantes no que se refere ao uso de termos matemáticos e ao desenvolvimento da ideia de coordenada, sem que houvesse correções ou discussões entre uma atividade e outra. Esses pontos serão especificados e discutidos a seguir.

No item (a), a maioria dos grupos respondeu corretamente em relação às alterações do gráfico, ou seja, relacionou a variável visual envolvida na atividade à unidade significativa valor para $n$ da expressão algébrica da função quadrática em sua forma canônica. No total foram dez (10) respostas corretas e três (03) parcialmente corretas. O Grupo 3 apresentou informações que não estavam corretas, escrevendo, por exemplo, que a parábola aumenta e fica abaixo do eixo x. O mesmo aconteceu com o Grupo 12. 
No item (b), os estudantes começaram a manifestar aproximações de notações de coordenadas cartesianas; em outras palavras, no lugar de indicar (incorretamente) um número para o vértice, passaram a representar um par de números, permitindo-lhes localizar o ponto e suas respectivas coordenadas no gráfico, conforme exemplo de respostas nas figuras 4 e 5 .

Figura 4 - Resolução do Grupo 8

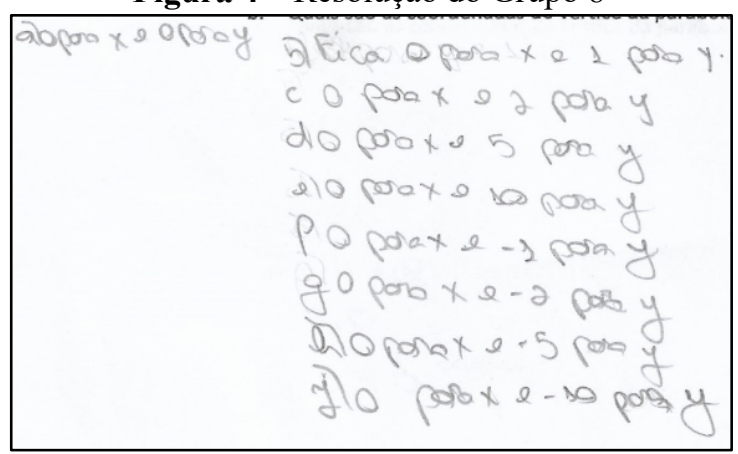

Fonte: dados da pesquisa.
Figura 5 - Resolução do Grupo 12

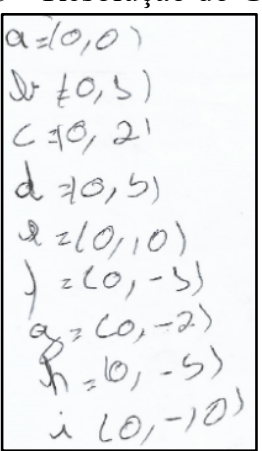

Fonte: dados da pesquisa.

Neste item, houve cinco (5) respostas corretas, seis (6) parcialmente corretas, e duas (2) incorretas. Comparando com o mesmo item na atividade anterior (zero [0] corretas, onze [11] parciais e duas [2] incorretas), observamos melhora no desempenho dos alunos no bloco de atividades 2 , principalmente no que diz respeito à notação de coordenada e ao emprego de termos matemáticos. A seguir, o quadro 5 apresenta o resumo das respostas dos estudantes para cada item desta atividade.

Quadro 5 - Resumo das respostas no Bloco de Atividades 2

\begin{tabular}{|c|c|c|l|}
\hline \multirow{2}{*}{ Grupo } & \multicolumn{2}{|c|}{$\begin{array}{c}\text { Situação por item } \\
\text { (C, P ou I) }\end{array}$} & \multicolumn{1}{c|}{ Apontamentos } \\
\cline { 2 - 3 } & (a) & (b) & \multicolumn{1}{|c|}{} \\
\hline 1 & C & P & b) Notação de coordenada. \\
\hline 2 & C & P & b) Incompleta. \\
\hline 3 & P & C & a) Informações incorretas. \\
\hline 4 & C & P & b) Notação de coordenada. \\
\hline 5 & C & P & b) Incompleta. \\
\hline 6 & P & P & $\begin{array}{l}\text { a) Incompleta; } \\
\text { b) Notação de coordenada. }\end{array}$ \\
\hline 7 & C & C & Sem apontamentos \\
\hline 8 & C & C & Sem apontamentos \\
\hline 9 & C & I & b) Não responde à pergunta. \\
\hline 10 & C & I & b) Não responde à pergunta. \\
\hline 11 & C & C & Sem apontamentos \\
\hline 12 & P & C & a) Informações incorretas. \\
\hline 13 & C & P & Sem apontamentos \\
\hline
\end{tabular}

Fonte: Autores. 


\section{Análise do Bloco de Atividades 3}

No bloco de atividades 3, para o estudo da variável visual posição do vértice com relação ao eixo das ordenadas, também observamos avanço no desempenho dos estudantes em relação às atividades anteriores. Notamos que os grupos utilizaram os termos matemáticos de modo mais coerente e suas produções escritas estavam mais claras.

Porém, mesmo com o avanço nas respostas dos grupos, alguns não conseguiram associar a variável visual envolvida na atividade com a unidade significativa correspondente na representação algébrica, como no caso do Grupo 4 (figura 6). Também a resposta Grupo 3, na figura 7, que apresenta indicativos de que o grupo não interpretou corretamente a questão.

Figura 6 - Resolução do Grupo $4^{6}$

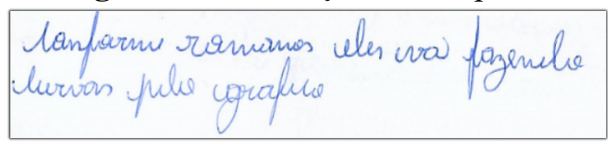

Fonte: dados da pesquisa.
Figura 7 - Resolução do Grupo 7 U concorridade do vertice lica para
cima sea for positivo (a)

Fonte: dados da pesquisa.

Para o item (a), identificamos seis (6) respostas corretas, uma (01) parcialmente correta e cinco (05) incorretas. Porém, notamos que houve um aprimoramento nas respostas dos grupos, pois no decorrer do processo de resolução das atividades, houve aprendizagem por parte dos estudantes no quesito de uso de termos matemáticos, como o uso do termo "parábola" que, nas primeiras respostas, como relatado, apareceu como "retas". Além do mais, ao término das atividades, as respostas dos alunos apresentam-se mais precisas e coerentes. A seguir, apresentamos o quadro 6, com o resumo das respostas dos alunos para esta atividade.

Quadro 6 - Resumo das respostas no bloco de atividades 3

\begin{tabular}{|c|c|c|l|}
\hline \multirow{2}{*}{ Grupo } & \multicolumn{2}{|c|}{$\begin{array}{c}\text { Situação por item } \\
\text { (C, P ou I) }\end{array}$} & \multirow{2}{*}{ Apontamentos } \\
\cline { 2 - 3 } & (a) & (b) & \\
\hline 1 & P & I & $\begin{array}{l}\text { a) Informações incorretas; } \\
\text { b) Não responde à pergunta. }\end{array}$ \\
\hline 2 & C & P & b) Notação de coordenada. \\
\hline 3 & I & P & $\begin{array}{l}\text { a) Não responde à pergunta; } \\
\text { b) Notação de coordenada. }\end{array}$ \\
\hline 4 & I & P & $\begin{array}{l}\text { a) Não responde à pergunta; } \\
\text { b) Notação de coordenada. }\end{array}$ \\
\hline 5 & I & - & $\begin{array}{l}\text { a) Não responde à pergunta; } \\
\text { b) Não fez. }\end{array}$ \\
\hline
\end{tabular}

\footnotetext{
6 “Conforme romanos, eles vão fazendo curvas pelo gráfico" (Transcrição da escrita dos alunos do Grupo 4 na Figura 8).
} 
Garcia, W. F. D. G.; Amplatz, L. C.; Rezende, V.

\begin{tabular}{|l|l|l|l|}
\hline 6 & C & - & b) Não fez. \\
\hline 7 & I & C & a) Não responde à pergunta. \\
\hline 8 & C & C & Sem apontamentos. \\
\hline 9 & C & I & b) Não responde à pergunta. \\
\hline 10 & C & C & Sem apontamentos. \\
\hline 11 & I & C & a) Não responde à pergunta. \\
\hline 12 & - & - & Não fez. \\
\hline 13 & C & P & $\begin{array}{l}\text { a) Não responde à pergunta; } \\
\text { b) Notação de coordenada. }\end{array}$ \\
\hline
\end{tabular}

Fonte: Autores.

\section{Análise do Bloco de Atividades 4}

O bloco de atividades 4 consiste em uma síntese das atividades anteriores. Notamos que a maioria dos estudantes, mesmo tendo respondido às atividades anteriores, não resolveram a atividade 4 na íntegra, e mencionaram apenas sobre a variação do coeficiente $a$. O quadro 7 contém a síntese das respostas dos estudantes para esta atividade.

Quadro 7 - Resumo das respostas no bloco de atividades 4

\begin{tabular}{|c|c|l|}
\hline Grupo & $\begin{array}{c}\text { Situação } \\
(\mathrm{C}, \mathrm{P} \text { ou I })\end{array}$ & \multicolumn{1}{|c|}{ Apontamentos } \\
\hline 1 & $\mathrm{P}$ & $\begin{array}{l}\text { Termos incorretos; } \\
\text { Incompleta. }\end{array}$ \\
\hline 2 & $\mathrm{C}$ & Sem apontamentos. \\
\hline 3 & $\mathrm{I}$ & Não responde à pergunta. \\
\hline 4 & $\mathrm{C}$ & Sem apontamentos. \\
\hline 5 & $\mathrm{I}$ & Não responde à pergunta. \\
\hline 6 & $\mathrm{P}$ & Incompleta. \\
\hline 7 & $\mathrm{C}$ & Sem apontamentos. \\
\hline 8 & $\mathrm{P}$ & Incompleta. \\
\hline 9 & $\mathrm{C}$ & Sem apontamentos. \\
\hline 10 & $\mathrm{P}$ & Incompleta. \\
\hline 11 & $\mathrm{P}$ & Incompleta. \\
\hline 12 & $\mathrm{P}$ & Incompleta. \\
\hline 13 & $\mathrm{P}$ & Incompleta. \\
\hline & $\mathrm{F} 0$ nte: & Autores. \\
\hline
\end{tabular}

Durante a implementação das atividades em sala de aula, notamos que os estudantes não estavam habituados com atividades que privilegiam a observação e a reflexão sobre objetos matemáticos. A princípio, demonstraram preocupação com fórmulas para calcularem os resultados e, quando mencionado sobre vértice da parábola 
na atividade 2, alguns grupos perguntaram a respeito da fórmula para o cálculo do " $\mathrm{x}$ vértice" e o "y vértice".

Essa preocupação por parte dos estudantes, em "resolver" as atividades com o uso de fórmulas, possivelmente surge pela priorização do tratamento no registro simbólico algébrico durante as aulas de Matemática, o qual é citado em diversas produções científicas, tais como Duval (2011) e Lucena e Gitirana (2016). Além disso, como citado anteriormente, Duval (2011) menciona que, em geral, no ensino de Matemática, apenas um sentido da conversão é priorizado, e no caso do ensino de funções, parte-se do registro de representação simbólico algébrico, passando pela representação numérica (tabular) para chegar na representação gráfica, por meio da abordagem ponto a ponto, fato que não contribui para a compreensão dos conceitos matemáticos em sua totalidade (DUVAL, 2011).

Notamos que os estudantes participantes desta pesquisa manifestaram dificuldades em escrever e explicitar suas observações, inclusive não utilizando os termos corretos, como "parábola", escrevendo, por exemplo, "reta" ou "curvas". Apesar dessas dificuldades, ao longo do desenvolvimento das atividades propostas nesta investigação, os estudantes passaram a escrever suas respostas de forma mais clara, organizadas e com notações matemáticas mais adequadas, ao serem comparadas com suas primeiras resoluções nas atividades do bloco 1 .

Além do mais, os estudantes perceberam que não era suficiente atribuir um valor numérico a um ponto e representá-lo apenas com uma das coordenadas.

\section{CONCLUSÕES}

A partir do desenvolvimento deste trabalho, notamos que o uso do software GeoGeobra, utilizado via Smartphones como instrumento para a aprendizagem em sala de aula, juntamente com as atividades propostas nesta pesquisa, as quais permitiram aos estudantes identificar as alterações nas variáveis visuais do gráfico e suas respectivas mudanças nas unidades significativas da expressão algébrica, proporcionaram, aos sujeitos colaboradores da pesquisa, estabelecer as conexões entre dois registros de representação semiótica - simbólico algébrico e gráfico - relativos a um mesmo objetivo matemático, a saber, função quadrática. Com isso, do ponto de vista de Duval (2011), as referidas atividades, aplicadas em sala de aula com o uso de smartphone e do aplicativo GeoGebra, proporcionam aos alunos aprendizagens do conceito matemático em questão. 
As análises desta pesquisa mostram que, a partir as atividades propostas aos estudantes, foi possível que os sujeitos colaboradores desta pesquisa notassem as variações dos gráficos de acordo com as variações na forma algébrica. Porém, isto só foi possível porque as atividades focaram na variação de uma unidade significativa algébrica de cada vez. Tal fato proporcionou aos alunos se apropriarem aos poucos das alterações realizadas no gráfico a cada modificação realizada na expressão algébrica, e vice e versa. Em outras palavras, com o decorrer da resolução das atividades, a cada variação das unidades significativas na representação algébrica, era possível que os estudantes observassem suas respectivas modificações nas variáveis visuais no gráfico da função quadrática. Esse fato vai ao encontro dos pressupostos de Duval (2011, p. 103) para a interpretação global de propriedades figurais, pois, para este pesquisador, em uma atividade, deve-se “[...] variar uma unidade significativa na expressão, mantendo as outras constantes e ver o que se passa no outro registro".

Durante as discussões das respostas com toda a turma, ao final da resolução dos blocos de atividades, foi possível observar que os estudantes haviam compreendido que as coordenadas do vértice da parábola estavam evidentes em sua forma algébrica, observando os gráficos no GeoGebra e fazendo essa conexão com a função, na sua forma algébrica. Além do mais, durante as discussões, os estudantes também manifestaram compreender os movimentos de translação horizontal e vertical de uma função quadrática, e sobre os respectivos vértices a partir da translação da função.

Por fim, podemos afirmar que os estudantes colaboradores desta pesquisa conseguiram compreender que tanto o registro de representação simbólico algébrico quanto o gráfico são representações do mesmo objeto matemático, bem como o significado de cada unidade significativa na expressão algébrica das funções quadráticas e sua implicação nas variáveis visuais do gráfico. Do ponto de vista de Duval (2011), esta articulação entre os registros gráfico e algébrico é um indicativo de que houve aprendizagens do conceito de função quadrática pelos dos estudantes.

Destacamos, ainda, a importância de se integrar, nas aulas de Matemática, as tecnologias disponíveis no dia a dia aos estudantes, pois, como visto nestas atividades, as resoluções dos estudantes e o uso do smartphone juntamente com o aplicativo GeoGebra possibilitaram estabelecer a conexão entre os registros gráfico e algébrico, por meio da abordagem de interpretação global das propriedades figurais (DUVAL, 2011), de modo preciso e ágil. Certamente os resultados não seriam os mesmos se as resoluções das 
atividades fossem realizadas apenas com os recursos lápis e papel, pois o dinamismo e a visualização simultânea das representações Algébrica e Gráfica, proporcionados pelo GeoGebra, são essenciais para a proposta de atividades e resultados adquiridos com esta pesquisa.

\section{REFERÊNCIAS}

BIRGIN, O. Investigation of Eighth-Grade Students' Understanding of the Slope of the Linear Function. Bolema. Rio Claro - SP, v. 26, n. 42A, p. 139-162, abr. 2012.

BRASIL. Ministério da Educação. Secretaria da Educação. Base Nacional Comum Curricular: Ensino Médio. Brasília: MEC, 2018.

DANTE, L. R. Matemática: Contexto \& Aplicações: ensino médio. 3. ed. São Paulo: Ática, v.1, 2017.

DUVAL, R. Registros de Representação Semióticas e Funcionamento Cognitivo da Compreensão em Matemática. In: MACHADO, S. D. A. Aprendizagem em Matemática: Registros de representação semiótica. Campinas: Papirus, 2003. p. 11-33.

DUVAL, R. Semiósis e pensamento humano: registros semióticos e aprendizagens intelectuais (fascículo I). Trad. de Lênio Fernandes Levy e Marisa Rosâni Abreu da Silveira. São Paulo: Livraria da Física, 2009.

DUVAL, R. Gráficos e equações: a articulação de dois registros. Tradução de Méricles Thadeu Moretti. Revemat. Florianópolis - SC. v. 6, n. 2, p. 96-112, 2011.

DUVAL, R. Registros de representação semiótica e funcionamento cognitivo do pensamento. Tradução de Méricles Thadeu Moretti. Revemat. Florianópolis - SC. v. 7, n. 2, p. 266-297, 2012.

FONSECA, V. G.; SANTOS, A. R.; NUNES, W. V. Estudo epistemológico do conceito de Funções: uma retrospectiva. In: XI Encontro Nacional de Educação Matemática., 2013, Curitiba. Anais do XI ENEM, 2013.

LUCENA, R.; GITIRANA, V. Articulações Internas à Matemática: a Parábola e a Função Quadrática com o GeoGebra. Educação Matemática em Revista, v. 21, n. 51, p. 25-34, 2016.

MAIA, D. Função Quadrática: Um estudo didático de uma abordagem computacional. 2007. 120 f. Dissertação (Mestrado em Educação Matemática) - Pontifícia Universidade Católica de São Paulo, São Paulo, 2007.

MORETTI, M. T. O papel dos Registros de Representação na aprendizagem de matemática. Contrapontos: Revista de Educação da Universidade do Vale do Itajaí. a. 2, n. 6, p. 343-362, Itajaí, set./dez. 2002.

MORETTI, M. T. A translação como recurso no esboço de curvas por meio da Interpretação Global de propriedades figurais. In: MACHADO, S. D.

A. Aprendizagem em Matemática: Registros de representação semiótica. Campinas: Papirus, 2003. p. 149-160.

NOGUEIRA, C. M. I. Construindo o conceito de funções. In: RAMOS, A. S.; REJANI, F. C. Teoria e Prática de Funções. Maringá: Centro Universitário de Maringá. Núcleo de Educação a Distância, 2014. 121 p. 
QUEIROZ, P. C. de. Uma proposta para o ensino de Função articulando as linguagens Algébrica e Geométrica. 2014. 158 f. Dissertação (Mestrado em Educação Matemática) - Universidade Federal do Mato Grosso do Sul, Campo Grande, 2014.

REIS, A. M. Uma proposta dinâmica para o ensino de função afim a partir de erros dos alunos no primeiro ano do ensino médio. 2011. 167 f. Dissertação (Mestrado Profissional em Ensino de Matemática) - Pontifícia Universidade Católica de São Paulo, São Paulo, 2011.

ROMANELLO, L. A. Potencialidades do uso do celular na sala de aula: atividades investigativas para o ensino de função. 2016. 135 f. Dissertação (Mestrado) Universidade Estadual Paulista, Instituto de Geociências e Ciências Exatas, Rio Claro, 2016.

SCANO, F. C. Função afim: uma sequência didática envolvendo atividades com o Geogebra. 2009. 149 f. Dissertação (Mestrado Profissional em Ensino da Matemática) Pontifícia Universidade Católica de São Paulo, São Paulo, 2009.

Submetido em 06 de agosto de 2020. Aprovado em 28 de novembro de 2020. 